\title{
Protective effect of berberine on doxorubicin-induced acute hepatorenal toxicity in rats
}

\author{
XUEYAN CHEN ${ }^{1,2^{*}}$, YU ZHANG ${ }^{3 *}$, ZHONGNING ZHU $^{1,2}$, HUANLONG LIU $^{4}$, HUICAI GUO ${ }^{1,2}$, \\ CHEN XIONG ${ }^{1,2}$, KERANG XIE ${ }^{1,2}$, XIAOFEI ZHANG ${ }^{1,2}$ and SUWEN SU ${ }^{1,2}$ \\ ${ }^{1}$ The Key Laboratory of Neural and Vascular Biology, Ministry of Education, Hebei Medical University; \\ ${ }^{2}$ The Key Laboratory of Pharmacology and Toxicology for New Drugs, Department of Pharmacology, \\ Hebei Medical University, Shijiazhuang, Hebei 050017; ${ }^{3}$ The Family Planning Research Institute of Hebei; \\ ${ }^{4}$ Pharmaceutical Department, The Second Hospital of Hebei Medical University, Shijiazhuang, Hebei 050000, P.R. China
}

Received March 19, 2015; Accepted January 21, 2016

DOI: $10.3892 / \mathrm{mmr} .2016 .5017$

\begin{abstract}
Doxorubicin (DOX), a potent broad-spectrum chemotherapeutic agent used for the treatment of several types of cancer, is largely limited due to its serious side effects on non-target organs. Thus, the present study aimed to investigate whether berberine (Ber), an isoquinoline alkaloid, could reduce DOX-induced acute hepatorenal toxicity in rats. Fifty rats were randomly divided into five groups: i) Control group, ii) DOX group, iii) DOX+Ber (5 mg kg) group; iv) DOX+Ber (10 mg kg), and v) DOX+Ber (20 mg kg) group. In the tests, body weight, organ index, general condition and mortality were observed. In addition, the serum levels of alanine transaminase (ALT), aspartate aminotransferase (AST), total cholesterol (TCHO) and blood urea nitrogen (BUN) were determined to evaluate hepatorenal function. Hepatorenal toxicity was further assessed using hematoxylin and eosin stained sections. Furthermore, the levels of superoxide dismutase (SOD), catalase (CAT), glutathione peroxidase (GPx) and malondialdehyde (MDA) in rat serum or tissue homogenate were also assessed to determine the mechanisms of action. Results suggested that pretreatment with Ber ameliorated the DOX-induced liver and kidney injury by lowering the serum ALT, AST, TCHO and BUN levels, and the damage observed histologically, such as hemorrhage and focal necrosis of liver and kidney tissues induced by DOX were also attenuated by Ber. Furthermore, Ber also exerted certain antioxidative properties through reversing the changes in the levels of MDA, SOD, GSH and MDA induced by DOX.
\end{abstract}

Correspondence to: Mrs. Suwen Su, The Key Laboratory of Pharmacology and Toxicology for New Drugs, Department of Pharmacology, Hebei Medical University, 361 East Zhongshan Road, Shijiazhuang, Hebei 050017, P.R. China

E-mail: suswmk@hebmu.edu.cn

${ }^{*}$ Contributed equally

Key words: doxorubicin, berberine, hepatorenal toxicity, oxidative damage
These findings indicate that Ber has protective effects against DOX-induced acute hepatorenal toxicity in rats. Combination of Ber with DOX is a novel strategy that has the potential for protecting against DOX-induced hepatorenal toxicity in clinical practice.

\section{Introduction}

Doxorubicin (DOX), an anthracycline antibiotic, is a potent anticancer drug commonly used in the treatment of numerous types of hemotological malignancy and solid tumors (1). However, the clinical application of DOX in cancer chemotherapy is largely limited by its serious side effects on non-target organs $(2,3)$. Despite the well-known side effects of DOX treatment associated with the heart, increasing evidence demonstrates that DOX also affects other organs, including the liver, kidney and brain $(4,5)$. Studies have also suggested that $\sim 40 \%$ of patients suffered from liver injury following treatment with doxorubicin (6). However, little is known regarding the effects and mechanisms of DOX toxicity on the liver and kidney system.

The exact mechanisms involved in DOX-induced cellular damage are complex and remain to be fully elucidated. A previous study demonstrated that oxidative stress was critical in the development of DOX-induced systemic injury (7). In the process of oxidative stress injury, excessive reactive oxygen species (ROS) are produced by DOX treatment, which is associated with cell death and is responsible for organ injury. It was reported that DOX predominantly caused liver injury via the generation of free radicals and the activation of nuclear factor- $\kappa \mathrm{B}$ (4). Therefore, effective strategies against DOX-induced oxidative stress injury would be expected to preserve or enhance the therapeutic effects of DOX in anticancer therapy. Thus far, to reduce the toxic effects of DOX, several pharmacologic agents, such as antioxidants, hematopoietic cytokines and iron-chelating agents have been investigated $(8,9)$. Although the majority of these agents have shown beneficial effects in terms of inducing oxidative stress, pharmacological and clinical attempts to reduce the hepatorenal toxicity of DOX have had little success thus far. Therefore, identification of novel effective strategies against DOX-induced complications is required. 
Berberine (Ber), an isoquinoline alkaloid, originally extracted from the traditional Chinese herb Coptis chinensis, is used for the treatment of bacterial infectious diseases, and has a long history for treating diarrhea in traditional Chinese medicine (10). Recently, an increasing number of studies have revealed that Ber displayed a wide range of pharmacological activities, such as reducing the risk of cancer, cardiovascular diseases and brain diseases due to its radical scavenging and antioxidant activities $(11,12)$. In addition, animal and clinical studies have suggested that Ber is beneficial in preventing reactive oxygen species (ROS) formation (13-15). In addition, it has been reported that Ber improved cardiac function in patients with severe congestive heart failure (16). Zhao et al (17) also reported that Ber may have a potential protective effect against DOX-induced cardiotoxicity. However, to the best of our knowledge, no study has been conducted concerning the protective effects of Ber on DOX-induced hepatorenal toxicity. Therefore, the present experiment was designed to investigate the possible protective effects of Ber on acute hepatorenal toxicity induced by DOX in rat model, and to further elucidated the mechanisms underlying these effects.

\section{Materials and methods}

Drugs and reagents. DOX was obtained from Sigma-Aldrich (St. Louis, MO, USA). Ber was obtained from Acros Organics (Geel, Belgium). Superoxide dismutase (SOD), malondialdehyde (MDA), catalase (CAT) and glutathione peroxidase (GPx) assay kits were all obtained from Jiancheng Bioengineering Institute (Nanjing, China). Alanine aminotransferase (ALT), aspartate aminotransferase (AST), blood urea nitrogen (BUN) and total cholesterol (TCHO) diagnostic kits were all obtained from Sysmex Corporation (Kobe, Japan). The paraffin, hematoxylin and eoisin were obtained from Beijing Solarbio Science \& Technology Co., Ltd. (Beijing, China).

Animals and experimental protocol. Male Sprague Dawley rats (experimental animal quality certificate no. 808046), weighing 250-300 g, were obtained from the experimental animal center of Hebei Medical University [license no. SCXK (Hebei) 2013-003]. The animals were housed under standard laboratory conditions with a $12 \mathrm{~h}$ light dark cycle at a $24 \pm 3^{\circ} \mathrm{C}$, and food and water provided ad libitum. Fifty rats were randomly divided into five groups: i) Control group; ii) DOX (20 mg kg) group; iii) DOX + Ber (5 mg kg) group; iv) DOX + Ber (10 mg kg); and v) DOX + Ber (20 mg kg) group. Ber was administered intragastrically once a day for 10 consecutive days in the DOX + Ber groups. Rats in groups the control and DOX groups received an equal volume of saline. DOX was injected intraperitoneally as a single dose (20 mg kg in saline) on the 8th day of Ber administration in the DOX and DOX + BER groups while an equal volume of saline in the control group. Mortality, general condition and body weight of the animals were observed during throughout the experiment. Then, on 10th day, $30 \mathrm{~min}$ after the final dose of Ber was administered, all rats were sacrificed with $80 \mathrm{mg}$ kg pentobarbital sodium (Merck Millipore, Dramstadt, Germany) via an intraperitoneal injection. The abdomen of each rat was opened, and blood samples were collected from the abdominal aorta. Next, the organs of liver and kidney were rapidly removed and washed with ice-cold saline, part of which was kept in liquid nitrogen and part of which was fixed in $4 \%$ paraformaldehyde. Subsequently, all analyses were performed according to the experimental protocol. The experiments were conducted in accordance with the Guide for Care and Use of Laboratory Animals published by the US National Institutes of Health, and were approved by the ethical committee for Animal Experiments.

Assessment of survival and general toxicity. The general condition, body weight and mortality of the experimental rats were recorded daily during throughout the experimental period. At the end of the experiment, all rats were anesthetized with $45 \mathrm{mg} \mathrm{kg}$ pentobarbital sodium via an intraperitoneal injection, and the abdomen of each rat was opened, then the fluid accumulation in the abdominal cavity was collected with a syringe and scored according to a graded scale of 0 to +++ (0: non; +: mild; ++: moderate and +++: severe) (18). Next, the organs of liver and kidney were removed and weighed, and the organ indexes were calculated according to the following formula: Organ index $=$ organ weight body weight.

Determination of serum biochemical indicators. Blood samples collected from the abdominal aorta were stored on ice prior to centrifugation at $2,100 \mathrm{xg}$ for $10 \mathrm{~min}$ within $1 \mathrm{~h}$ of collection. Then, the supernatant was collected for the determination of biochemical indicators. The levels of ALT, AST, TCHO and BUN in the serum were determined as sensitive indicators of liver damage, according to the manufacturer's protocol of diagnostic kits with a CHEMIX-180 automatic biochemistry analyzer (Sysmex Corporation).

Histopathological analysis. At the end of the study period, the liver and kidney were excised from all study animals, and sections were fixed in $4 \%$ formaldehyde directly after excision. The tissues were then dehydrated in an ascending series of ethanol (70, 80, 96 and 100\%). Following paraffin embedding, transverse sections with a thickness of $5 \mu \mathrm{m}$ were stained with hematoxylin-eosin, and histological examination was conducted using a light microscope (Olympus BX-50 Olympus Corporation, Tokyo, Japan).

Determination of lipid peroxides. The preparation of serum was conducted as described. The liver or kidney tissues were homogenized in ice-cold saline to form a $10 \%$ homogenate. The homogenate was then centrifuged at 2,100 $\mathrm{x} g$ for $15 \mathrm{~min}$ at $4^{\circ} \mathrm{C}$, then the supernatants were collected and the total protein content was detected with a BCA Protein Assay kit (Pierce; Thermo Fisher Scientific, Inc., Waltham, MA, USA). Next, the levels of SOD, CAT, GPx and MDA in the serum and tissue homogenate were determined using a commercially available assay kits according to the manufacturer's protocol.

Statistical analysis. All values are presented as the mean \pm standard deviation. Statistical analyses were performed using one-way analysis of variance, a $\chi^{2}$ was used for the count data and Dunnett's test (SPSS for Windows v11.0, SPSS Inc., Chicago, IL, USA). P $<0.05$ was considered to indicate a statistically significant difference. 
Table I. Influence of Ber on DOX-induced abdominal, pleural and pericardial effusion intensity score in surviving rats.

\begin{tabular}{|c|c|c|c|c|c|c|c|c|c|}
\hline \multirow[b]{3}{*}{ Group } & \multirow[b]{3}{*}{$\mathrm{n}$} & \multicolumn{8}{|c|}{ Effusion intensity score } \\
\hline & & \multicolumn{2}{|c|}{0} & \multicolumn{2}{|c|}{+} & \multicolumn{2}{|c|}{++} & \multicolumn{2}{|c|}{+++} \\
\hline & & $\mathrm{N}$ & $\%$ & $\mathrm{~N}$ & $\%$ & $\mathrm{~N}$ & $\%$ & $\mathrm{~N}$ & $\%$ \\
\hline Con & 10 & 10 & 100 & 0 & 0 & 0 & 0 & 0 & 0 \\
\hline DOX & 8 & 0 & 0 & 0 & 0 & 0 & 0 & 8 & $100^{\mathrm{a}}$ \\
\hline Ber $5 \mathrm{mg} / \mathrm{kg}$ & 10 & 0 & 0 & 4 & 40 & 3 & 30 & 3 & $30^{\mathrm{a}, \mathrm{b}}$ \\
\hline Ber $10 \mathrm{mg} / \mathrm{kg}$ & 10 & 1 & 10 & 4 & 40 & 2 & 20 & 3 & $30^{\mathrm{a}, \mathrm{b}}$ \\
\hline Ber 20 mg/kg & 10 & 1 & 10 & 5 & 50 & 2 & 33.3 & 2 & $20^{\mathrm{a}, \mathrm{b}}$ \\
\hline
\end{tabular}

Score: $(0)$ none; (+) mild; (++) moderate; $(+++)$ severe. Statistical evaluation was performed using the $\chi^{2}$-test. ${ }^{a} \mathrm{P}<0.01$ vs. Con. ${ }^{\text {b }}<0.01$ vs. DOX.

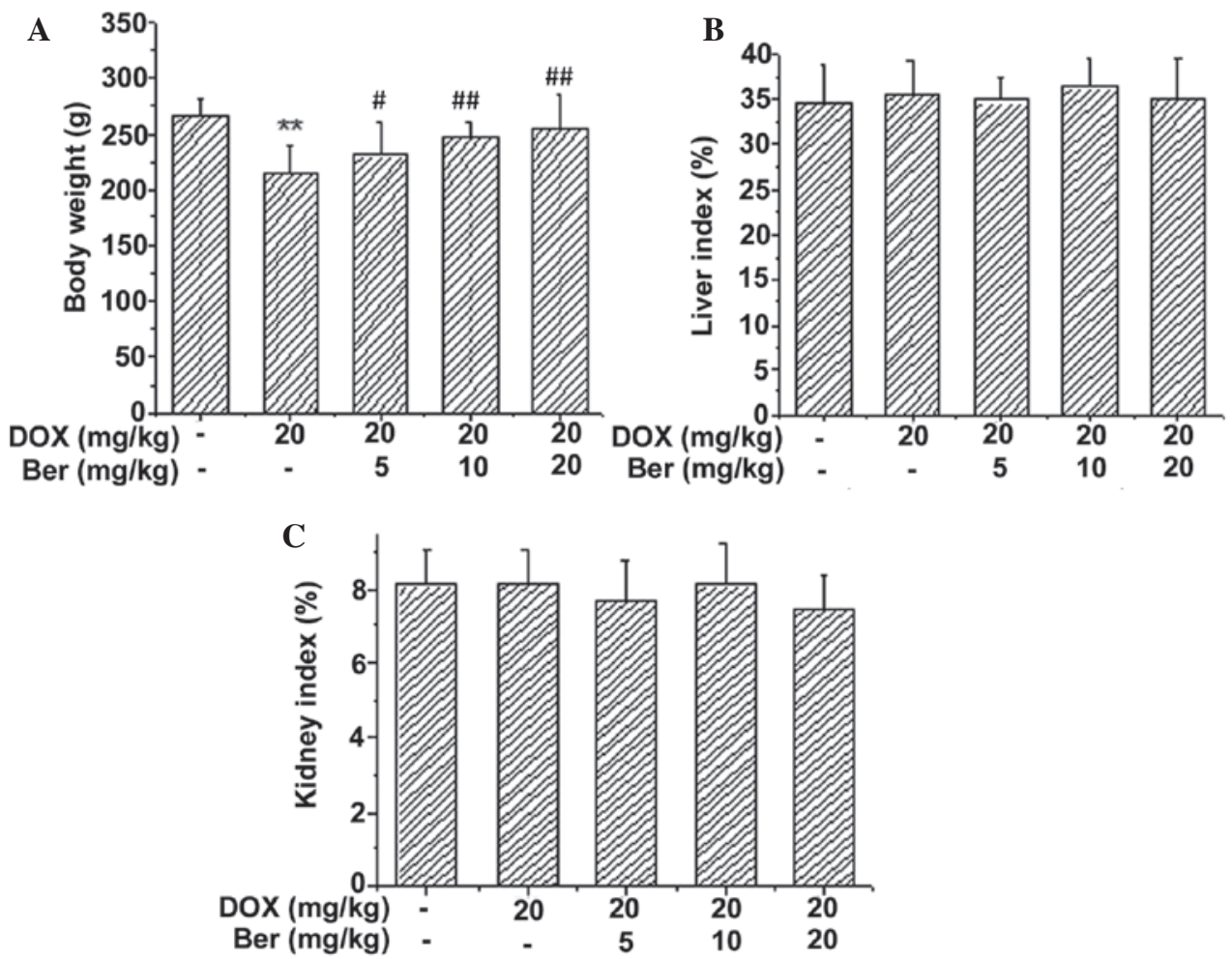

Figure 1. Effects of Ber on (A) body weight, (B) liver index and (C) kidney index in DOX-induced acute injury in rats ( $\mathrm{n}=8$-10). Values are expressed as the mean \pm standard deviation. ${ }^{* *} \mathrm{P}<0.01$ vs. the control group, and ${ }^{\#} \mathrm{P}<0.05,{ }^{\# \#} \mathrm{P}<0.01$ vs. the DOX-treated group. DOX, doxorubicin; Ber, berberine.

\section{Results}

Effects of Ber on survival and general toxicity in DOX-induced acute injury in rats. The general condition, body weight and mortality of the experimental rats were recorded daily throughout the experimental period. Two rats died in the DOX-treated group. However, no fatalities were observed in any of the other groups. Moreover, reduced appetite, decreased activity and progressive physical exhaustion were observed in the rats from the DOX-treated group (data not shown). These animals also presented with scruffy and a light yellow-tinged fur compared to the control and Ber+DOX-treated group. The body weight and organ indexes $30 \mathrm{~min}$ after the last Ber dose was administered (day 10) are shown in Fig. 1. It was demonstrated that DOX treatment resulted in reduced body weights compared with the control group $(\mathrm{P}<0.01)$. The body weight was significantly increased in the Ber-treated groups $(5,10$ and $20 \mathrm{mg} \mathrm{kg}$ ) compared with the DOX group $(\mathrm{P}<0.05, \mathrm{P}<0.01$ and $\mathrm{P}<0.01$, respectively) (Fig. 1A). However, Fig. $1 \mathrm{~B}$ and $\mathrm{C}$ suggested that there was no significant difference in the liver and kidney indexes among the five groups.

Notably, the rats in the DOX-alone group were also observed to develop ascites, as determined by a grossly distended abdomen and later confirmed during necropsy. At necropsy, the most prominent gross pathological change in the rats treated with DOX was the presence of excessive pericardial, pleural 


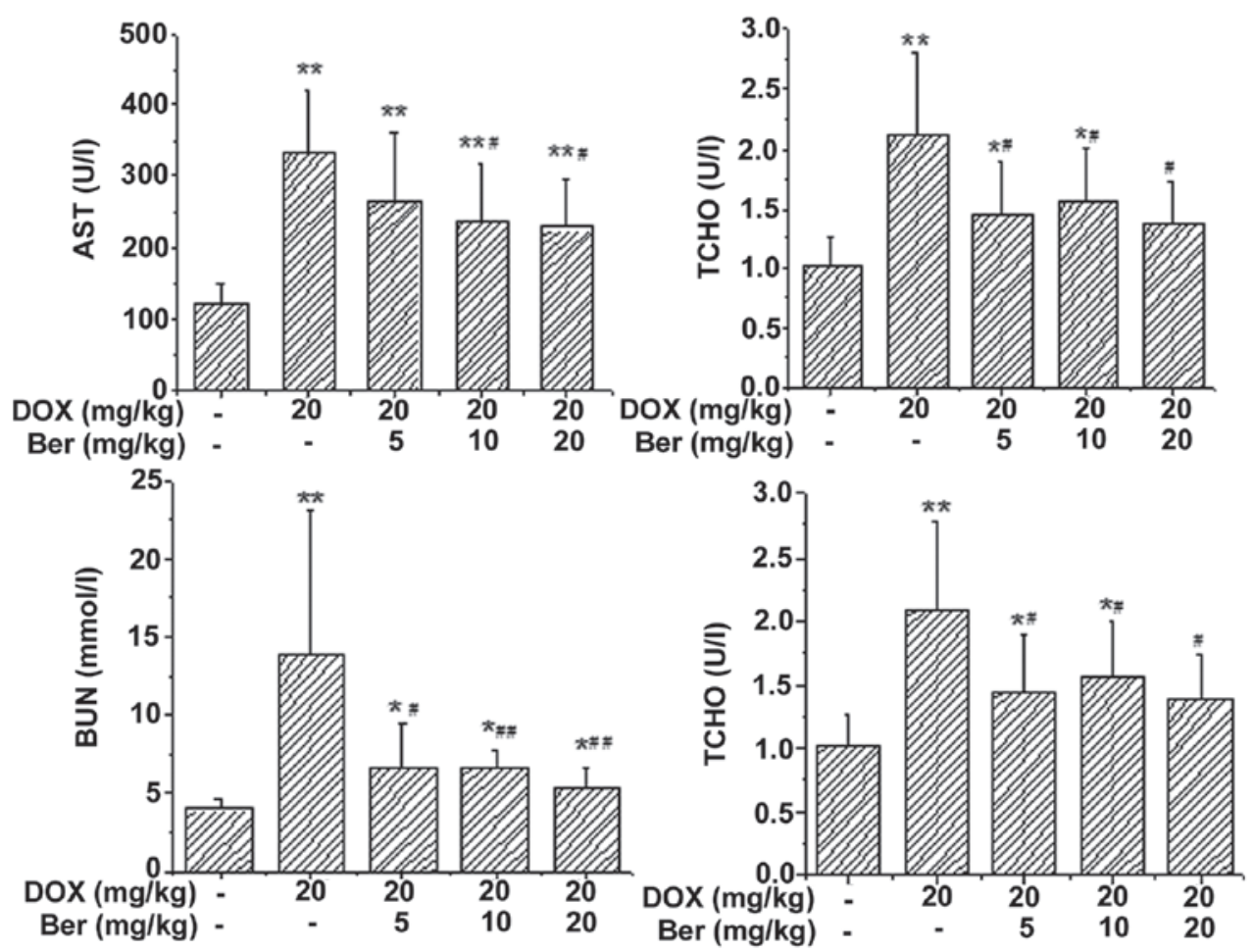

Figure 2. Effects of Ber on the activity of ALT, AST, TCHO and the content of BUN in the serum of rats with DOX-induced acute injury. Values are presented as the mean \pm standard deviation. " $\mathrm{P}<0.05,{ }^{* *} \mathrm{P}<0.01$ vs. the control group, and ${ }^{\#} \mathrm{P}<0.05,{ }^{\# \prime} \mathrm{P}<0.01$ vs. the DOX-treated group. Ber, berberine; ALT, alanine transaminase; AST, aspartate aminotransferase; TCHO, total cholesterol; DOX, doxorubicin.
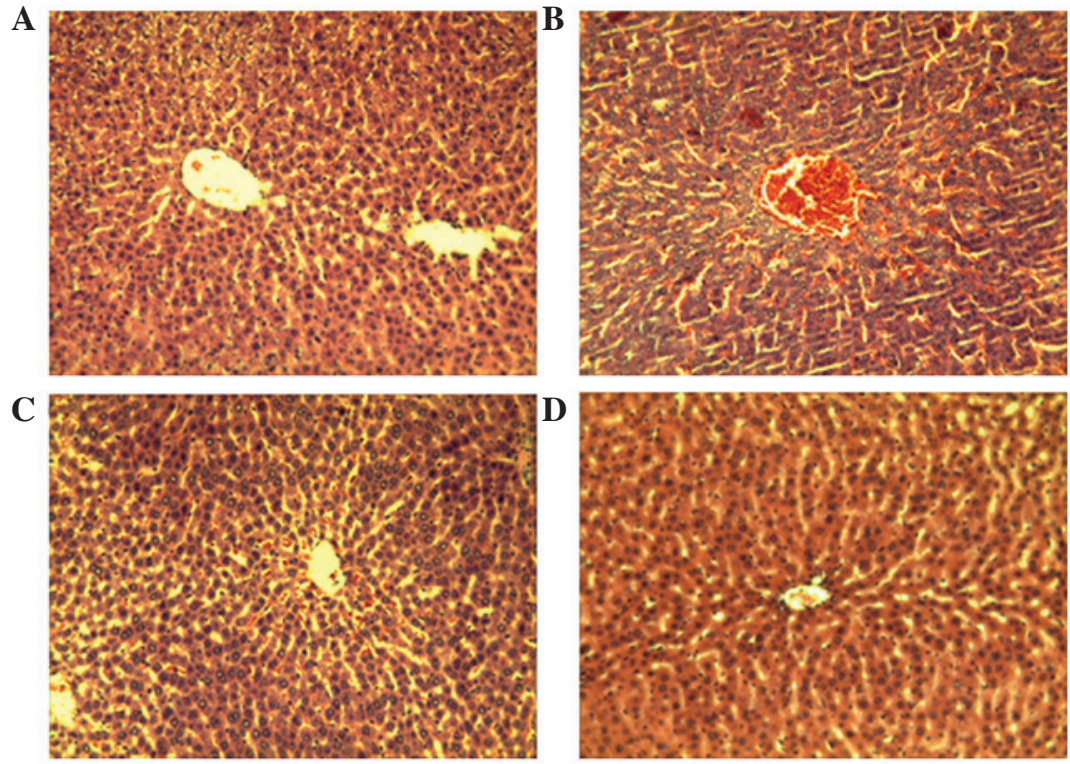

Figure 3. Representative histopathological section obtained from the livers of different groups (stain, hematoxylin and eosin; magnification, x200). (A) Control group, normal structure of hepatic lobule and central vein; (B) DOX group, passive congestion in central vein and liver sinusoid are pronounced; (C) Ber $(5 \mathrm{mg} / \mathrm{kg})$ group, passive congestion in central vein and liver sinusoid are alleviated; (D) Ber $(20 \mathrm{mg} / \mathrm{kg})$ group, primarily normal hepatic tissue structure. Ber, bereberine; DOX, doxorubicin.

and peritoneal fluid. The effusion intensity score was severe in $100 \%$ of the rats in the DOX-treated group compared with $0 \%$ of the control group $(\mathrm{P}<0.01)$. However, treatment with Ber significantly decreased the amount of pericardial, pleural and peritoneal fluids. Compared with the DOX-treated group, the effusion intensity score was improved in the three Ber-treated groups $(5,10,20 \mathrm{mg} \mathrm{kg})(\mathrm{P}<0.01$; Table I).
Effects of Ber on the serum levels of ALT, AST, TCHO and $B U N$. The serum levels of ALT, AST, TCHO and BUN have been widely used clinically as parameters for the diagnosis of hepatorenal diseases. As shown in Fig. 2, DOX alone induced significant increases in serum ALT, AST, TCHO and BUN levels compared with the control group $(\mathrm{P}<0.01)$, and these increases were effectively attenuated by treatment with 5 , 

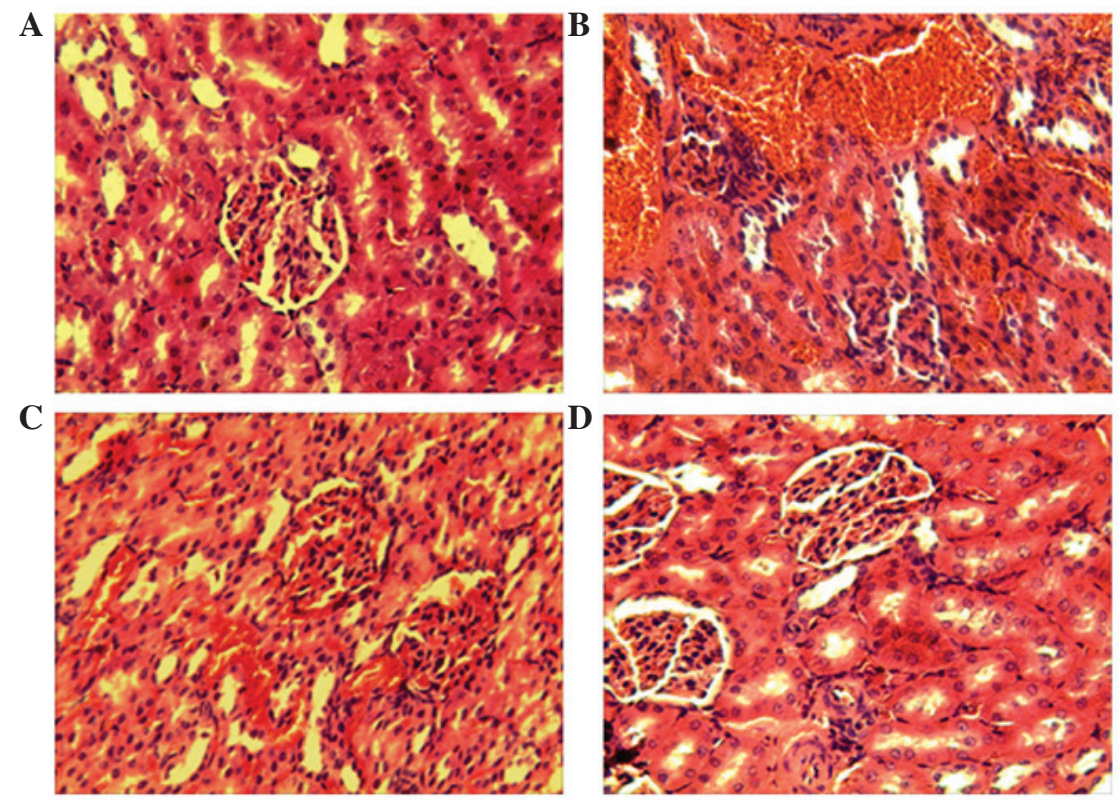

Figure 4. Representative histopathological sections obtained from the kidney tissue in different groups (stain, hematoxylin and eosin; magnification, x200). (A) Control group, normal structure of the glomerulus and tubules; (B) DOX group, tubulointerstitial lesions and bleeding; (C) Ber (5 mg/kg) group, alleviatived hyperemic glomerulus and tubulointerstitial lesions; (D) Ber (20 mg/kg) group, primarily normal structure of kidney tissue. Ber, berberine; DOX, doxorubicin.

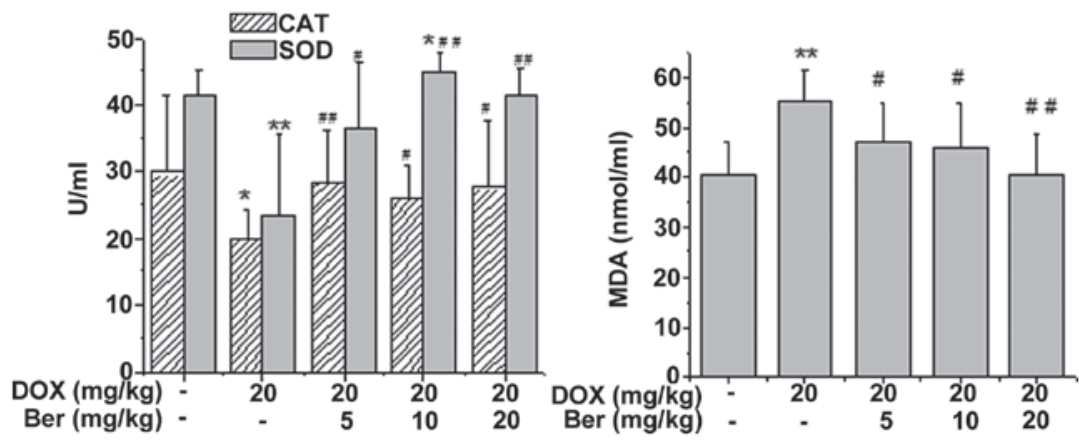

Figure 5. Effects of Ber on the activity of CAT, SOD and the content of MDA in the serum of rats with DOX-induced acute injury. Values are expressed as the mean \pm standard deviation. ${ }^{*} \mathrm{P}<0.05,{ }^{* *} \mathrm{P}<0.01$ vs. the control group, ${ }^{\#} \mathrm{P}<0.05,{ }^{\# \#} \mathrm{P}<0.01$ vs. the DOX-treated group. DOX, doxorubicin; Ber, berberine; CAT, catalase; SOD, superoxide dismutase; MDA, malondialdehyde.

10 and $20 \mathrm{mg} \mathrm{kg}$ Ber $(\mathrm{P}<0.05$ or $\mathrm{P}<0.01)$. However, although these levels declined following treatment with Ber, they did not return to the baseline levels. These results suggest that Ber effectively protects the liver and kidney against DOX-induced hepatorenal toxicity.

Histopathological examination. Hepatorenal toxicity induced by DOX in rats was further assessed using hematoxylin and eosin stained sections. Representative examples of the histological appearance in the control, DOX-treated and Ber+DOX-treated groups are shown in Figs. 3 and 4. Fig. 3 shows the histopathological changes of the rat liver. The liver sections from control group showed regular cell distribution and normal integrated structures of liver lobules (Fig. 3A). Following administration of DOX the liver exhibited notable histopathological change. DOX induced tissue injuries, including degeneration of the hepatocytes, focal necrosis and hemorrhage. Moreover, visible congestion and expansion also appeared in the central veins. (Fig. 3B). By contrast, the severe hepatic injury induced by DOX was alleviated by Ber treatment (Fig. 3C and D).
Representative examples of the histological appearance of rat kidney are shown in Fig. 4. The microscopic examination for the kidney in the control group revealed normal histology parameters (Fig. 4A). Whereas, kidney tissues from the DOX-treated group showed widespread structural abnormalities, with congestion in glomerular tissues and irregular hemorrhages in the renal interstitial (Fig. 4B). By contrast, the severe histopathological changes in the kidney induced by DOX was limited by Ber treatment (Fig. 4C and D).

Ber alleviates DOX-induced oxidative damage. To determine whether Ber alleviated DOX-induced oxidative damage in rats, the SOD, CAT and GPx activity, as well as the content of MDA in rat serum or tissue homogenate, were assessed. As shown in Fig. 5, DOX treatment led to a significant increase in the content of MDA and a manifest depletion in the activity of CAT and SOD in the serum compared with that of the control group $(\mathrm{P}<0.05$ or $\mathrm{P}<0.01)$. Whereas, Ber $(5,10$ and $20 \mathrm{mg} \mathrm{kg})$ treatment notably reversed the DOX-induced changes in the MDA, CAT and SOD levels in the rat serum $(\mathrm{P}<0.05$ or $\mathrm{P}<0.01)$. 

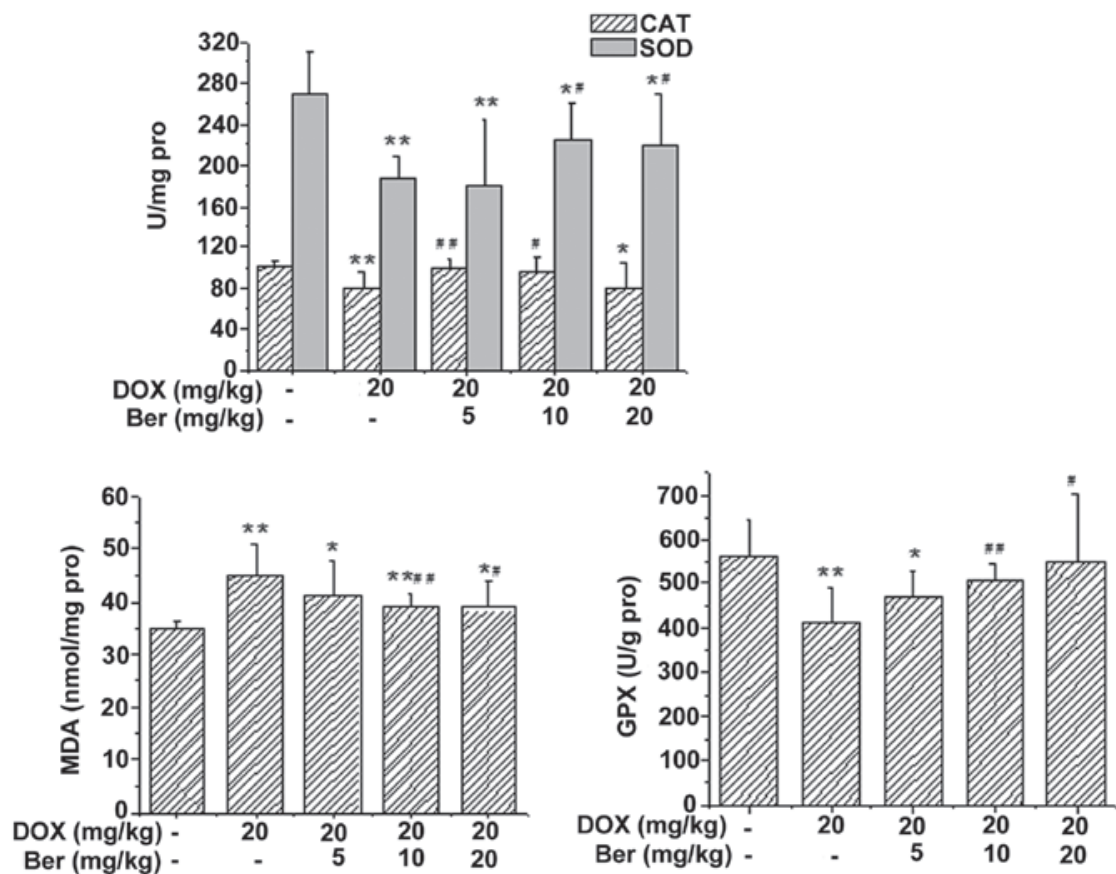

Figure 6. Effects of Ber on the activity of CAT, SOD, GPx and the content of MDA in the hepatic tissue of DOX-treated rats. Values are expressed as the mean \pm standard deviation. ${ }^{~} \mathrm{P}<0.05,{ }^{* *} \mathrm{P}<0.01$ vs. the control group, ${ }^{\#} \mathrm{P}<0.05,{ }^{\# \#} \mathrm{P}<0.01$ vs. the DOX-treated group. CAT, catalase; SOD, superoxide dismutase; MDA, malondialdehyde; GPx, glutathione peroxidase; DOX, doxorubicin; Ber, berberine.
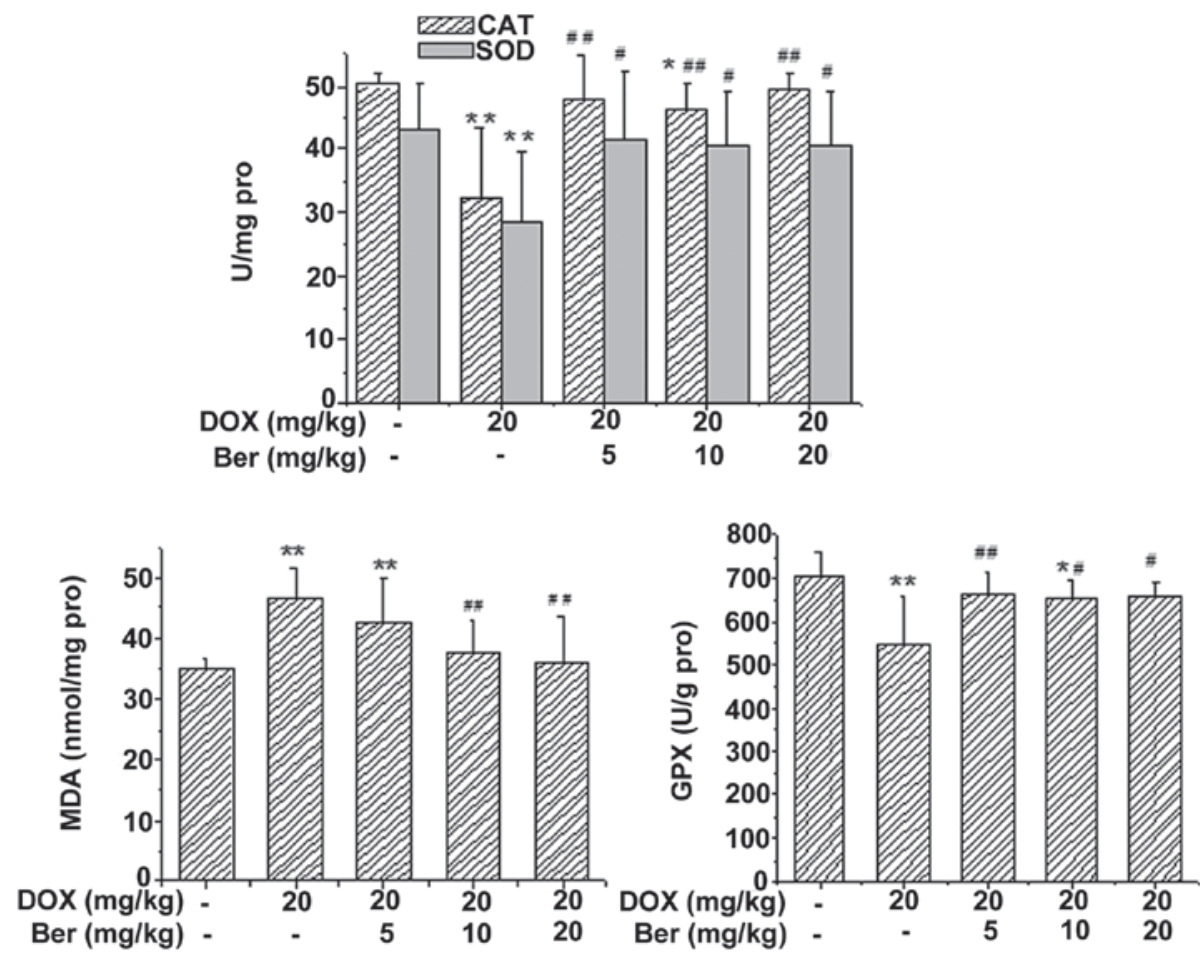

Figure 7. Effects of Ber on the activity of CAT, SOD and GPx, and the content of MDA in the renal tissue of DOX-treated rats. Values are expressed as the mean \pm standard deviation. " $\mathrm{P}<0.05,{ }^{*} \mathrm{P}<0.01$ vs. the control group, ${ }^{\#} \mathrm{P}<0.05,{ }^{\# \#} \mathrm{P}<0.01$ vs. the DOX-treated group. CAT, catalase; SOD, superoxide dismutase; MDA, malondialdehyde; GPx, glutathione peroxidase; DOX, doxorubicin; Ber, berberine.

In addition, the activity of SOD, CAT and GPx, and the content of MDA in rat tissue homogenate were also assessed. As shown in Figs. 6 and 7, DOX treatment led to a significant increase in the content of MDA and a manifest depletion in the activity of GPx, CAT and SOD in the liver and kidney tissue homogenate compared with that of the control group $(\mathrm{P}<0.05$ or $\mathrm{P}<0.01)$. Whereas, Ber (10 and $20 \mathrm{mg} \mathrm{kg}$ ) treatment reversed the DOX-induced changes in the MDA, SOD and GPx levels in rat liver tissue homogenate $(\mathrm{P}<0.05$ or $\mathrm{P}<0.01)$, and Ber $(5$ and $10 \mathrm{mg} \mathrm{kg}$ ) also reversed the DOX-induced reduction in the 
activity of CAT ( $\mathrm{P}<0.05$ or $\mathrm{P}<0.01$, Fig. 6). Additionally, in rat kidney tissue homogenate, $\operatorname{Ber}(5,10,20 \mathrm{mg} \mathrm{kg})$ significantly increased the activity of CAT, SOD and GPx compared with that of the DOX-treated group $(\mathrm{P}<0.05$ or $\mathrm{P}<0.01$, Fig. 7). The elevated content of MDA induced by DOX was also decreased by $\operatorname{Ber}(10$ and $20 \mathrm{mg} \mathrm{kg})$ treatment $(\mathrm{P}<0.01$, Fig. 7$)$.

\section{Discussion}

With the wide application of chemotherapeutic drugs in tumor therapy, adverse reactions that limit the dosage and duration of treatment, and affect the quality of life have gained attention. Therefore, it is important to develop effective parts of Chinese herbal medicine against the adverse reactions of chemotherapeutic drugs. The present study demonstrated that Ber, an isoquinoline alkaloid, originally extracted from the traditional Chinese herb Coptis chinensis, suppressed the hepatorenal toxicity induced by DOX administration. Pretreatment with Ber ameliorated the DOX-induced liver and kidney injury by lowering the serum levels of ALT, AST, TCHO and BUN, and the histological damage, such as hemorrhage and focal necrosis of the liver and kidney tissues induced by DOX were also attenuated by Ber. This suggested that Ber may exhibit a protective role in DOX-induced hepatorenal toxicity, and may be a potential agent for attenuation and prevention of the serious complications of DOX in clinical practice.

DOX has been reported to be a potent and effective anticancer agent (19). However, the therapeutic application of DOX has been greatly limited by its dose-dependent toxicity, particularly severe cardiac and hepatic toxicity $(20,21)$. DOX poisoning is usually divided into acute toxicity, subacute toxicity and chronic toxicity categories. Acute poisoning commonly occurs following single use or after a period of treatment, with the most common symptoms including hypotension, arrhythmia and cardiac dysfunction may occur occasionally, and often accompanied by hepatic and renal damage $(22,23)$. In the current study of DOX toxicity, the single high-dose model is widely used, which provides valuable biological insights into DOX-induced organ injury. In the single high-dose model, the dosage is equivalent to a high-dose single injection in cancer patients, similar to the experimental design implemented in the present study.

Concurrent with clinical trials on DOX-induced organ injury, the present study showed that the significant increase in the activity of AST, ALT, TCHO, BUN, and the histopathological changes in the liver and kidney were observed in the DOX treatment group. These biochemical and pathological alterations in the rat model resemble the acute liver and kidney failure observed in humans (24). ALT and AST are the enzymes required in the mutual transformation of sugar and protein in the body. ALT predominantly exists in liver cells, and AST is mainly located in myocardial cells; however, the serum level of AST is also increased when the liver is damaged; thus, the increase in the serum level of ALT and AST suggests liver damage. Higher serum aminotransferase activity may be the result of leakage from damaged liver cell membranes following DOX treatment (25). BUN is the major end metabolic product of human protein, and is one of the main indexes for the evaluation of renal function. Therefore, the increase in these biochemical indexes suggest that DOX causes acute damage of the liver and kidney. Moreover, DOX-induced hepatorenal toxicity was further evaluated by analyzing the histopathology.

Since DOX has significant antitumor activity, novel methods to reduce or prevent its detrimental side effects are expected to increase its effectiveness in anticancer therapy. The traditional Chinese medicine Rhizoma Coptidis has also been demonstrated to exhibit notable antitumor effects (26). In Rhizoma Coptidis and its active ingredients, Ber is the most valuable active component, and exhibits antidiabetic, antitumor, antibacterial and anti-inflammatory effects $(12,27)$. Therefore, it was hypothesized that Ber may have synergistic antitumor effects with DOX, and relieve the systemic toxicity induced by DOX. Tong et al (26) revealed that Ber treatment potentiated the sensitivity of cancer cells to DOX. It was also reported that Ber suppressed tumor growth through the induction of apoptosis and cell cycle arrest in cancer cells (28). The present study was designed to investigate the potential protective effects of Ber against DOX-induced hepatorenal toxicity in rats. In the current study, DOX administration resulted in increased mortality rates compared with control rats. Live rats showed excessive pericardial, pleural and peritoneal effusion when treated with DOX. However, pretreatment with Ber showed protective effects against DOX-induced mortality and effusion intensity score. Moreover, Ber also protected the liver and kidney function by lowering serum ALT, AST, TCHO and BUN levels. Furthermore, the protective effect of Ber against DOX was also evaluated histopathologically. The histological damages such as congestion, necrosis and severe destruction of hepatic architecture induced by DOX were also markedly attenuated by Ber pretreatment. These findings indicated that Ber exhibited a potential protective agent against DOX injury.

However, the mechanism underlying the effects of Ber is unclear. Further research is required to identify with precision the protective effect of Ber, and the exact mechanism of Ber on DOX-induced liver and kidney injury. Some hypotheses as to the mechanism underlying the effect of Ber have been reported, such as free radical scavenging ability, antiapoptotic and anticarcinogenic actions $(29,30)$. Ber is an effective antioxidant and free radical scavenger which can prevent ROS formation and produce protective effects on cardiac function (31). Additionally, animal and clinical investigations showed that Ber was beneficial in combating against ROS formation $(32,33)$. Whereas, it is still unknown whether the protective effect of Ber on hepatic and renal function was due to its antioxidative properties. Thus, it is reasonable to investigate the antioxidant status of Ber in DOX-induced acute hepatorenal toxicity in rats. In the present study, the antioxidant activity of Ber was revealed through the oxidative markers as well as antioxidant enzymes. The oxidative marker MDA is a product of lipid peroxidation which is released during oxidative stress. SOD, CAT and GPx act as the antioxidant defense system of the body (34). Current data showed that the MDA levels in serum and hepatorenal tissues were significantly elevated. Moreover, the activity of antioxidant enzymes CAT, SOD and GPx was significantly reduced following DOX administration. Whereas, Ber significantly decreased the DOX-induced elevation of MDA, and increased the activity of CAT, SOD and GPx as compared with the DOX group. The findings of the present study suggested that elevated lipid peroxidation, accompanied by deteriorating antioxidant status, was evident in the DOX group. Pretreatment of rats 
with Ber significantly alleviated the oxidative stress induced by DOX. Thus, Ber protected the DOX-induced hepatic and renal injury due to its antioxidative properties.

In conclusion, the present study reported the protective effect of Ber against DOX-induced acute hepatorenal toxicity in a rat model. Pretreatment with Ber significantly inhibited DOX-induced liver and kidney dysfunction and histopathological changes. The mechanism underlying the effects of Ber is likely to be through attenuating the oxidative stress injury. This suggests that Ber may have a role in the attenuation and prevention of the serious complications of DOX. Thus the combination of Ber with DOX is a novel strategy that has the potential for protecting against DOX-induced hepatorenal toxicity in clinical practice.

\section{Acknowledgements}

This study was supported by the Natural Science Foundation of China (grant nos. 81273600 and 81302773) and the natural science Foundation of Hebei Province (grant nos. C2011206145 and $\mathrm{H} 2013206147)$.

\section{References}

1. Cheng C, Xue W, Diao H, Xia S, Zuo L, He A, Gao F, Huang Z, Chen $\mathrm{J}$ and Zhang J: Antitumor activity and toxicological properties of doxorubicin conjugated to [alpha], [beta]-poly [(2-hydroxyethyl)-L-aspartamide] administered intraperitoneally in mice. Anticancer Drugs 21: 362-371, 2010.

2. Tangpong J, Miriyala S, Noel T, Sinthupibulyakit C, Jungsuwadee P and St Clair DK: Doxorubicin-induced central nervous system toxicity and protection by xanthone derivative of Garcinia mangostana. Neuroscience 175: 292-299, 2011.

3. Cardoso S, Santos RX, Carvalho C, Correia S, Pereira GC, Pereira SS, Oliveira PJ, Santos MS, Proenca T and Moreira PI: Doxorubicin increases the susceptibility of brain mitochondria to $\mathrm{Ca}(2+)$-induced permeability transition and oxidative damage. Free Radic Biol Med 45: 1395-1402, 2008.

4. Kalender Y, Yel M and Kalender S: Doxorubicin hepatotoxicity and hepatic free radical metabolism in rats. The effects of vitamin $\mathrm{E}$ and catechin. Toxicology 209: 39-45, 2005.

5. Bárdi E, Bobok I, Voláh A, Kappelmayer J and Kiss C: Anthracycline antibiotics induce acute renal tubular toxicity in children with cancer. Pathol Oncol Res 13: 249-253, 2007.

6. Yang XL, Fan CH and Zhu HS: Photo-induced cytotoxicity of malonic acid [C(60)]fullerene derivatives and its mechanism. Toxicol In Vitro 16: 41-46, 2002.

7. Badkoobeh P, Parivar K, Kalantar SM, Hosseini SD and Salabat A Effect of nano-zinc oxide on doxorubicin-induced oxidative stress and sperm disorders in adult male Wistar rats. Iran J Reprod Med 11: 355-364, 2013.

8. Li L, Takemura G, Li Y, Miyata S, Esaki M, Okada H, Kanamori H, Khai NC, Maruyama R, Ogino A, et al: Preventive effect of erythropoietin on cardiac dysfunction in doxorubicin-induced cardiomyopathy. Circulation 113: 535-543, 2006.

9. Yeh YC, Lai HC, Ting CT, Lee WL, Wang LC, Wang KY, Lai HC and Liu TJ: Protection by doxycycline against doxorubicin-induced oxidative stress and apoptosis in mouse testes. Biochem Pharmacol 74: 969-980, 2007.

10. Tang LQ, Wang FL, Zhu LN, Lv F, Liu S and Zhang ST: Berberine ameliorates renal injury by regulating $\mathrm{G}$ proteins-AC- cAMP signaling in diabetic rats with nephropathy. Mol Biol Rep 40: 3913-3923, 2013.

11. Wang Y, Huang Y, Lam KS, Li Y, Wong WT, Ye H, Lau CW, Vanhoutte PM and Xu A: Berberine prevents hyperglycemia-induced endothelial injury and enhances vasodilatation via adenosine monophosphate-activated protein kinase and endothelial nitric oxide synthase. Cardiovasc Res 82: 484-492, 2009.

12. Derosa G, Maffioli P and Cicero AF: Berberine on metabolic and cardiovascular risk factors: An analysis from preclinical evidences to clinical trials. Expert Opin Biol Ther 12: 1113-1124, 2012.
13. Cho BJ, Im EK, Kwon JH, Lee KH, Shin HJ, Oh J, Kang SM, Chung JH and Jang Y: Berberine inhibits the production of lysophosphatidylcholine-induced reactive oxygen species and the ERK1 2 pathway in vascular smooth muscle cells. Mol Cells 20: 429-434, 2005.

14. Li J, Pan Y, Kan M, Xiao X, Wang Y, Guan F, Zhang X and Chen L: Hepatoprotective effects of berberine on liver fibrosis via activation of AMP-activated protein kinase. Life Sci 98: 24-30, 2014.

15. Hsu YY, Tseng YT and Lo YC: Berberine, a natural antidiabetes drug, attenuates glucose neurotoxicity and promotes Nrf2-related neurite outgrowth. Toxicol Appl Pharmacol 272: 787-796, 2013.

16. Marin-Neto JA, Maciel BC, Secches AL and Gallo Junior L: Cardiovascular effects of berberine in patients with severe congestive heart failure. Clin Cardiol 11: 253-260, 1988.

17. Zhao X, Zhang J, Tong N, Liao X, Wang E, Li Z, Luo Y and Zuo H: Berberine attenuates doxorubicin-induced cardiotoxicity in mice. J Int Med Res 39: 1720-1727, 2011.

18. Kelishomi RB, Ejtemaeemehr S, Tavangar SM, Rahimian R, Mobarakeh JI and Dehpour AR: Morphine is protective against doxorubicin-induced cardiotoxicity in rat. Toxicology 243: 96-104, 2008.

19. Jing L, Wu Y, Wu J, Zhao J, Zuo D and Peng S: Peroxiredoxins are involved in metallothionein protection from doxorubicin cardiotoxicity. Eur J Pharmacol 659: 224-232, 2011.

20. Hou XW, Jiang Y, Wang LF, Xu HY, Lin HM, He XY, He JJ and Zhang S: Protective role of granulocyte colony-stimulating factor against adriamycin induced cardiac, renal and hepatic toxicities. Toxicol Lett 187: 40-44, 2009.

21. You JS, Pan TL and Lee YS: Protective effects of Danshen (Salvia miltiorrhiza) on adriamycin-induced cardiac and hepatic toxicity in rats. Phytother Res 21: 1146-1152, 2007.

22. Outomuro D, Grana DR, Azzato F and Milei J: Adriamycin-induced myocardial toxicity: New solutions for an ole problems? Int J Cardiol 117: 6-15, 2007.

23. Piscitelli SC, Rodvold KA, Rushing DA and Tewksbury DA: Pharmacokinetics and pharmacodynamics of doxorubicin in patients with small-cell lung cancer. Clin Pharmacol Ther 53: 555-561, 1993.

24. Swain SM, Whaley FS and Ewer MS: Congestive heart failure in patients treated with doxorubicin: A retrospective analysis of three trials. Cancer 97: 2869-2879, 2003.

25. King SL, Mohiuddin JJ and Dekaney CM: Paneth cells expand from newly created and preexisting cells during repair after doxorubicin-induced damage. Am J Physiol Gastrointest Liver Physiol 305: G151-G162, 2013.

26. Tong N,Zhang J, Chen Y,Li Z, Luo Y, Zuo H and Zhao X: Berberine sensitizes mutliple human cancer cells to the anticancer effects of doxorubicin in vitro. Oncol Lett 3: 1263-1267, 2012.

27. Abd El-Wahab AE, Ghareeb DA, Sarhan EE, Abu-Serie MM and El Demellawy MA: In vitro biological assessment of Berberis vulgaris and its active constituent, berberine: Antioxidants, anti-acetylcholinesterase, anti-diabetic and anticancer effects. BMC Complement Altern Med 13: 218, 2013.

28. Yan K, Zhang C, Feng J, Hou L, Yan L, Zhou Z, Liu Z, Liu C, Fan Y, Zheng $\mathrm{B}$ and $\mathrm{Xu} \mathrm{Z}$ : Induction of G1 cell cycle arrest and apoptosis by berberine in bladder cancer cells. Eur J Pharmacol 661: 1-7, 2011.

29. Youn MJ, So HS, Cho HJ, Kim HJ, Kim Y, Lee JH, Sohn JS, Kim YK, Chung SY and Park R: Berberine, a natural product, combined with cisplatin enhanced apoptosis through a mitochondria caspase-mediated pathway in HeLa cells. Biol Pharm Bull 31: 789-795, 2008.

30. Hsu YY, Chen CS, Wu SN, Jong YJ and Lo YC: Berberine activates Nrf2 nuclear translocation and protects against oxidative damage via a phosphatidylinositol 3-kinase Akt-dependent mechanism in NSC34 motor neuron-like cells. Eur J Pharm Sci 46: 415-425, 2012.

31. Lv XX, Yu XH, Wang HD, Yan YX, Wang YP, Lu DX, Qi RB, Hu CF and Li HM: Berberine inhibits norepinephrine-induced apoptosis in neonatal rat cardiomyocytes via inhibiting ROS-TNF- $\alpha$-caspase signaling pathway. Chin J Integr Med 19: 424-431, 2013.

32. Cheng F, Wang Y, Li J, Su C, Wu F, Xia WH, Yang Z, Yu BB, Qiu YX and Tao J: Berberine improves endothelial function by reducing endothelial microparticles-mediated oxidative stress in humans. Int J Cardiol 167: 936-942, 2013.

33. Shen L and Ji HF: The mechanisms of ROS-photogeneration by berberine, a natural isoquinoline alkaloid. J Photochem Photobiol B 99: 154-156, 2010.

34. Zhao X, Zhang J, Tong N, Chen Y and Luo Y: Protective effects of berberine on doxorubicin-induced hepatotoxicity in mice. Biol Pharm Bull 35: 796-800, 2012. 